Angelo Vanzulli, MD - Alessandro DelMaschio, MD • Pierluigi Paesano, MD • Francesca Braggion, MD

Chiara Livieri, MD • Enzo Angeli, MD • Gabriele Tomasi, MD • Carlo Gatti, MD

Francesca Severi, MD • Giuseppe Chiumello, MD

\title{
Testicular Masses in Association with Adrenogenital Syndrome: US Findings ${ }^{1}$
}

Adrenogenital syndrome (AGS) is the result of inborn enzymatic defects in the synthesis of steroid hormones. The production of cortisol is deficient and that of adrenocorticotropic hormone is increased. Sometimes male patients have clinically detectable testicular lesions, known as testicular tumors of AGS (TTAGS). From 1985 to 1991, scrotal ultrasonography (US) was performed in 30 consecutive pubertal and postpubertal patients with AGS to investigate the prevalence and US characteristics of TTAGS. Eight of 30 patients had a testicular lesion (27\%); six of the eight lesions were clinically undetected. The mean diameter of the lesions was $16.44 \mathrm{~mm}$ (range, 2-28 mm). The lesions were hypoechoic in all cases, with welldefined margins in six cases. The nodules were multifocal in all patients and bilateral in six $(\mathbf{7 5 \%})$. If testicular lesions are present in a patient with AGS, TTAGS are likely, and frequent US monitoring is adequate for diagnostic evaluation.

Index terms: Adrenal gland, abnormalities, 86.143 - Testis, neoplasms, 847.3 - Testis, US, 847.1298

Radiology 1992; 183:425-429

' From the Department of Radiology (A.V., A.D., P.P., E.A.) and the Pediatric Clinic (F.B., G.C.), Scientific Institute St Raffaele, University Hospital, Olgettina 60, 20132 Milan, Italy; the Pediatric Clinic, University of Pavia, Scientific Institute Policlinico St Matteo, Pavia, Italy (C.L., F.S.); and the Department of Radiology, Fondazione Clinica Del Lavoro, Pavia, Italy (G.T.

C.G.). From the 1990 RSNA scientific assembly. Received September 13, 1991; revision requested October 29; revision received December 18; accepted December 30 . Address reprint requests to A.V.

RSNA, 1992
AREnOgenital syndrome (AGS) is $A$ the result of enzymatic defects that are inherited as autosomal recessive traits and result in deficient production of cortisol and the accumulation of hormonal precursors (1). The frequency of AGS is approximately one in 14,000 births (2). The most common hormonal defect is a deficiency of 21-hydroxylase, which occurs in about $90 \%$ of the cases. While 11-hydroxylase and 17-hydroxylase deficiencies have also been observed, they are rare $(5 \%$ and less than $1 \%$ of cases, respectively) (3) (Fig 1). Deficiency of 21-hydroxylase has been described as occurring in three forms: a classic form with prenatal virilization with or without salt wasting, a late-onset form, and an asymptomatic form (3).

Male patients can have testicular lesions that manifest clinically, at puberty or later, as an increase in the size of the testis, often with a nodular pattern (4). These lesions are known as testicular tumors of AGS (TTAGS) (4). TTAGS are often bilateral and can decrease in size when treated with adequate hormonal therapy. Histologically, TTAGS consist of focal areas of endocrine cells that become hypertrophied after prolonged stimulation with adrenocorticotropic hormone (ACTH). It has been hypothesized that the endocrine cells involved in TTAGS resemble Leydig cells morphologically but behave like adrenocortical cells (5). These testicular lesions can be a sign of poor hormonal control, with the need for more aggressive treatment (4).

While there are some clinical and pathologic descriptions of these testicular lesions in the literature, to our knowledge there are only a few articles describing their ultrasound (US) characteristics $(6,7)$. The frequency of TTAGS in a population of living patients with AGS is unknown. In one study none of the 52 men evaluated had clinically detectable testicular nodules (8), whereas in another study all 14 men had histologic evidence of TTAGS (9). These testicular masses may pose diagnostic difficulties, especially when AGS is not considered, and testicular biopsy and even orchiectomy may be performed unnecessarily.

The aims of the present study were to evaluate the clinical behavior of these lesions, including patient age at onset and tumor growth rates, as well as the sonographic appearance of the TTAGS.

\section{MATERIALS AND METHODS}

From January 1985 through June 1991, we studied 30 consecutive male pubertal or postpubertal patients with AGS (age range, 9-32 years; mean, 17.8 years). The clinical and laboratory data for these patients are summarized in Figure 2. Biochemical confirmation of AGS (the criterion for selection of patients) consisted of elevated serum levels of testosterone and 17-hydroxyprogesterone and elevated urinary excretion of 17-ketosteroids and pregnanetriol for the patients with 21-hydroxylase deficiency; 11-hydroxylase deficiency was confirmed with assays of desoxycorticosterone and 11-cortisol.

Hormone control was assessed with evaluation of assays of $(a)$ 17-OH progesterone and $(b)$ adrenocorticotropic hormone $(\mathrm{ACTH})$ and findings of $(c)$ bone age and $(d)$ growth charts. Hormone control was classified as good, fair, or poor (1).

All 30 patients were examined with scrotal ultrasound (US). For patients in whom no abnormality was seen on the initial scan, US scanning was repeated annually. If the initial scan revealed pathologic changes, the patient underwent US scanning every 3-6 months. US follow-up of the patients ranged from 1 to 6 years (mean, 3 years 1 month). Commercially available US equipment was used (Toshiba Medical Systems, Tokyo; GE Medical Systems, Milwaukee; and Ansaldo Medical

Abbreviations: $\quad \mathrm{ACTH}=$ adrenocorticotropic hormone, $\mathrm{AGS}=$ adrenogenital syndrome, TTAGS $=$ testicular tumors of AGS. 
Systems, Genua, Italy) with high-frequency linear probes $(7.5 \mathrm{MHz})$. Scrotal US scanning was performed in the longitudi nal, axial, and oblique planes, with use of codified methods (10). A silicone pad (Kitecko-3M, Malakoff, Cedex, France) was applied to the scrotum to optimize visualization of the superficial portions of the testes. US examinations were always performed by a staff radiologist (P.P., C.G.) with experience in pediatric radiology and pediatric US. The presence, shape, number, and echogenicity of the lesions were recorded and whether the lesions were uni- or bilateral. Hard-copy scans were obtained for further analysis; the images were retrospectively reviewed by two examiners (A.V., P.P.) in conference.

Findings at clinical and imaging follow-up were considered the standard of reference for the study. To differentiate between testicular changes related to AGS and to testicular malignancy, we considered the decrease in size or number of the lesions after hormonal therapy to be evidence of TTAGS. If the lesions were multifocal and showed either no change or slight increase in size, follow-up of at least 1 year was considered sufficient to rule out a neoplasm.

\section{RESULTS}

US depicted testicular lesions in eight of 30 patients with AGS (27\%) (Fig 2). In only two of these eight patients were the abnormalities clinically palpable. The mean diameter of the testicular lesions was $16.44 \mathrm{~mm}$ (range, 2-28 mm). The clinical, biochemical, and US data of the eight subjects with TTAGS are summarized in the Table. Two patients of the group with TTAGS (patients 1 and 2, who were brothers) had 11-hydroxylase deficiency. The other six patients, who were not related, had 21-hydroxylase deficiency. The age at detection of testicular lesions varied from 10 to 21 years (mean, 15.6 years). The mean age of the 22 patients without testicular lesions at US was 17 years (range, 9-32 years). Hormonal control was poor in four of the eight patients (50\%) with TTAGS, compared with control in six of 22 patients $(27 \%)$ without testicular lesions at US.

The nodules were always multifocal $(100 \%)$ and often bilateral (patients $1-6[75 \%])$. The location was intratesticular in all cases; one patient also had two apparently extratesticular lesions, in the epididymis (Fig 3). The testicular lesions revealed at US were always hypoechoic but only mildly hypoechoic in three cases. In one case a hyperechoic rim was detected (Fig 4). The margins were well defined in six cases (Fig 5) and were blurred in the other two.
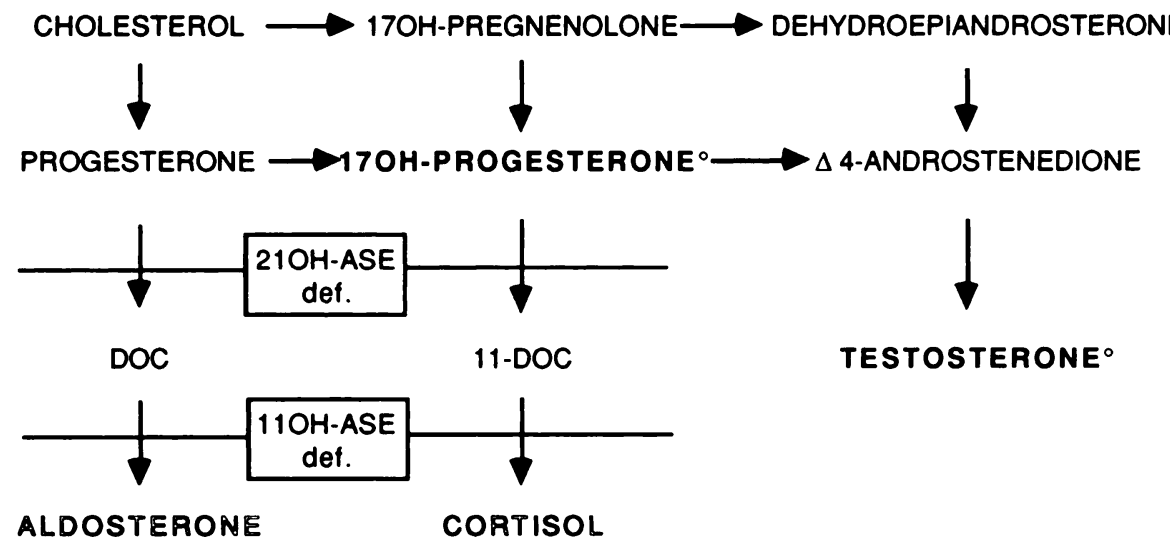

Figure 1. Flow chart depicts metabolic pathway of the synthesis of aldosterone and cortisol. Enzyme defects are in evidence ${ }^{\circ}=$ hormonal precursors that accumulate, $\Delta=$ hormone, $\operatorname{def}=$ deficiency, $O H-A S E=\mathrm{OH}$ hydroxylase.

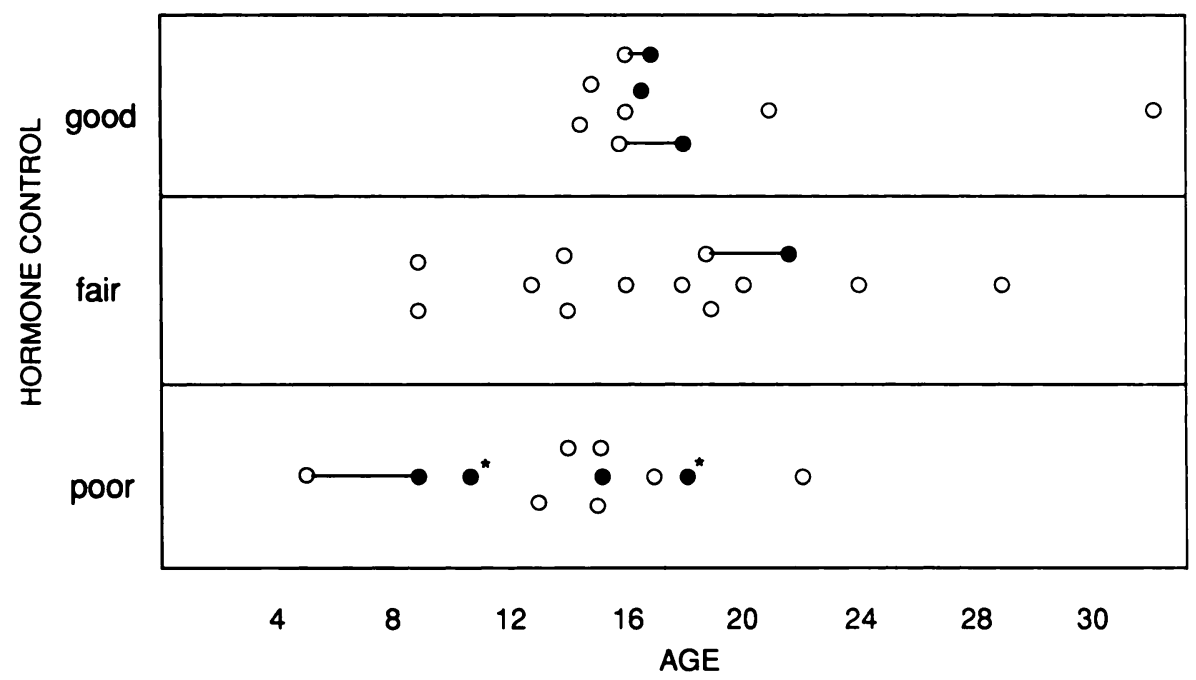

Figure 2. Graph depicts hormone control on the basis of clinical and laboratory data of the 30 patients. $\mathrm{O}=$ normal testicles at US, $O=$ TTAGS, $\mathrm{O}-\mathrm{O}=$ normal findings on initial US scan that became positive in the follow-up US scan, ${ }^{*}=$ patients with $11-\mathrm{OH}$ hydroxylase deficiency.

The characteristics of the eight patients who had positive US findings for TTAGS include the following data. Patient 1 was first examined in 1987, when he was 24 years old. At that time, bilateral multifocal lesions with a clinically palpable testicular nodular pattern were seen at US. Findings from a previous US examination performed at another institution in 1982 (when the patient was 18 years old) were of bilateral nodular lesions. Patient 2 underwent his first US examination in 1981, at another institution, when the boy was 11 years old; findings in this examination were reported to be positive. In 1987 a US examination performed at our institution confirmed the presence of bilateral hypoechoic lesions in the testes and the epididymis (Fig 3).

Patient 3 underwent scrotal US scanning at another institution in
1982; findings were reported as normal. In 1987, when he was 10 years old, a US scan depicted bilateral hypoechoic foci located near the testicular hilum. These abnormalities did not change in the 4 years of follow-up (Fig 6). Patients 4 and 5 underwent their first US examinations in 1988 (at ages 16 and 15 years, respectively). TTAGS were diagnosed in each. Patient 4 was the only one in whom the follow-up US scans revealed an increase in the size of the lesions (from $19 \mathrm{~mm}$ to $21 \mathrm{~mm}$ in 3 years). Biopsy was not performed of these lesions because this small increase in size was not considered significant. Hormonal control was good in this patient.

Findings at US in patient 6 were negative in January 1989 (when the patient was 16 years old). One year later bilateral testicular involvement was apparent clinically and at US. 


\begin{tabular}{|c|c|c|c|c|c|c|c|c|c|}
\hline $\begin{array}{c}\text { Patient } \\
\text { No. }\end{array}$ & $\begin{array}{l}\text { Age (y) } \\
\text { at First } \\
\text { Positive } \\
\text { US Scan }\end{array}$ & $\begin{array}{l}\text { Age (y) } \\
\text { at Prior } \\
\text { Negative } \\
\text { US Scan* }\end{array}$ & $\begin{array}{c}\text { TTAGS } \\
\text { Clinically } \\
\text { Present }\end{array}$ & $\begin{array}{c}\text { Hormonal } \\
\text { Control }\end{array}$ & $\begin{array}{l}\text { Range in Size } \\
\text { of Tumor } \\
\text { (mm) }\end{array}$ & Bilateral & Multifocal & $\begin{array}{c}\text { Years of } \\
\text { Follow-up }\end{array}$ & $\begin{array}{l}\text { Findings at } \\
\text { Follow-up } \ddagger\end{array}$ \\
\hline $1^{+}$ & 18 & NA & Yes & Poor & $7-25$ & Yes & Yes & 5.0 & No change \\
\hline $2^{\dagger}$ & 11 & NA & No & Poor & $14-20$ & Yes & Yes & 6.0 & No change \\
\hline 3 & 10 & 5 & No & Poor & $10-20$ & Yes & Yes & 4.0 & No change \\
\hline 4 & 16 & NA & No & Good & $11-19$ & Yes & Yes & 3.0 & $\begin{array}{l}\text { Mild increase in } \\
\text { diameter (to } \\
21 \mathrm{~mm} \text { ) }\end{array}$ \\
\hline 5 & 15 & NA & No & Poor & $2-6$ & Yes & Yes & 3.0 & No change \\
\hline 6 & 17 & 16 & Yes & Good & $26-28$ & Yes & Yes & 1.5 & $\begin{array}{l}\text { Mild decrease in } \\
\text { diameter (to } \\
18 \mathrm{~mm} \text { ) }\end{array}$ \\
\hline 7 & 21 & 19 & No & Fair & $3-4$ & No & Yes & 1.5 & No change \\
\hline 8 & 17 & 16 & No & Good & $3-5$ & No & Yes & 1.0 & No change \\
\hline
\end{tabular}

* NA = not applicable.

+ Patients with 11-OH hydroxylase deficiency.

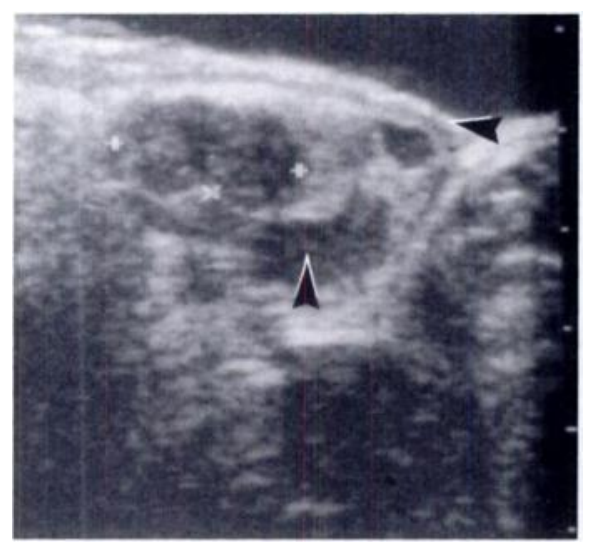

Figure 3. Patient 2. Oblique sonogram shows lesions (cursors) in both the testis and the tail of the epididymis (arrowheads).

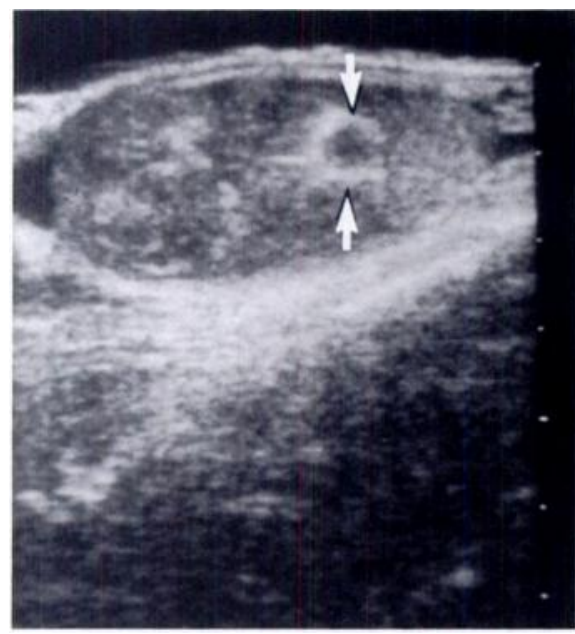

Figure 4. Patient 1. Longitudinal sonogram of the right testis shows multifocal hypoechoic lesions with blurred margins and echogenic rim (arrows).

During follow-up for $1 \frac{1}{2}$ years, the lesions decreased in size after treatment with appropriate hormonal therapy. At the patient's last US examination, the diameter of the lesions had decreased from $26 \times 9 \mathrm{~mm}$ to $18 \times 6 \mathrm{~mm}$ and from $28 \times 9 \mathrm{~mm}$ to $17 \times 7 \mathrm{~mm}$.

Two of the eight patients (patients 7 and 8 [25\%]) had unilateral multiple lesions. In patient 7 testicular US scans were negative from 1987 through 1989 , but multiple small ( $<4$-mm-diameter) hypoechoic lesions were detected at US in one testis in 1990, when the patient was 21 years old. Findings at US examinations were negative for the 1st year in patient 8; in the 2 nd year, when the patient was 17 years old, US examination revealed two small hypoechoic foci ( $<5 \mathrm{~mm}$ in diameter) in the right testis.

\section{DISCUSSION}

The most common inborn enzymatic defect that causes AGS is 21hydroxylase deficiency, which has been described as existing in three forms: a classic form with prenatal virilization with or without salt wasting, a late-onset form, and an asymptomatic form. Other enzyme defects are rare and sometimes do not result in virilization (1).

Some pubertal or postpubertal male patients with AGS have clinically detectable testicular masses that must be differentiated from malignancy (4) because they are completely benign, with no malignant potential. It has been known for 40 years that testicular "tumors" occur in patients with AGS (11), but there is considerable controversy about the origin of these tumorlike lesions. Although some authors have called the lesions ectopic adrenal tissues (11), others believe that they represent Leydig cells (12). The biochemical resemblance of the tumor cells to those of the associated hyperplastic adrenal cortex lends sup- port to the theory of origin in adrenal cortical rests. Adrenal cortical rests, found in the vicinity of the testis in $7.5 \%-15.0 \%$ of newborn (13) and $1.6 \%$ of adult testes (14), are predominantly extratesticular (13). This location is a finding that supports the argument against the rests giving rise to TTAGS, since TTAGS are intratesticular in $95 \%$ of cases (15). In our experience, all lesions were intratesticular, except in patient 2 , who also had extratesticular lesions.

Regardless of the origin, TTAGS are caused by elevated levels of ACTH, and similar testicular lesions can be found in patients with other conditions, such as Addison disease and Cushing syndrome, that are also characterized by elevated levels of ACTH (6). TTAGS may pose diagnostic difficulties both clinically and pathologically. If AGS is not taken into account, a testicular mass is usually assumed to be a neoplasm, and an orchiectomy may be performed (4). At other times the diagnosis of AGS may not be made until a testicular mass develops; in a study of 40 patients with AGS this was the case in seven patients $(18 \%)$ (15). At histologic examination the lesions may be misinterpreted to be a Leydig cell tumor (15). In 1953 Prader demonstrated that the testicular masses are not autonomous neoplasms but are dependent on the elevated level of ACTH that occurs in patients with AGS and that the masses decrease in size if the level of ACTH is lowered by the administration of corticosteroids (16).

It is difficult to establish on clinical grounds the frequency of testicular tumors in patients with AGS. In one study none of 52 adult male patients had testicular nodules (8), whereas nine of 17 male patients $(53 \%)$ were 
found to have testicular nodules at clinical examination in another study (17). The prevalence appears to be related to hormonal control and to the age of the patients: The lesions that were detected clinically developed during periods of inadequate hormonal therapy as a result of low dosage or poor compliance (4). In a recent investigation (7), however, scrotal US scanning revealed testicular lesions in seven of $15(47 \%)$ male patients with AGS who were not in poor hormonal control. Clinical palpation revealed testicular changes in six of these patients.

The purposes of the present study were to assess the frequency of TTAGS in a population of living AGS patients who were screened with US and to describe the sonographic patterns of the lesions. The prevalence of TTAGS in our study was $27 \%$. No patient had the late-onset form of AGS. The majority of TTAGS were discovered in patients between the ages of 10 and 20 years (Fig 1). Normal US scans had been obtained in those patients as recently as 1 year prior to observation of the TTAGS, indicating that annual surveillance is probably appropriate during this period. Negative findings at US do not preclude future development of the lesions, but once the lesions develop they are relatively slow growing. In our study, four of the eight patients with testicular lesions had poor hormonal control, but only two had clinical evidence of the lesions and in one of these (patient 6) hormonal control was good (Table). On the other hand, only $27 \%$ of the patients without TTAGS depicted at US were in poor hormonal control. These data suggest that testicular nodules detected at US occur frequently and that poor compliance with hormonal therapy is found in approximately one-half of the patients with testicular nodules.

The diameter of the lesions in patient 4 increased slightly in the follow-up period. The patient complied well with treatment, and the increase could not be attributed to poor hormonal control. The increase might have been the result of partial autonomy of the nodule, and the patient is now examined more frequently. Patient 6 was treated aggressively with steroids, and the lesions decreased in diameter from approximately $3 \mathrm{~cm}$ to less than $2 \mathrm{~cm}$ in 1 year. The lesions in the other six patients did not change in size during follow-up, despite hormonal control that was poor in four patients and good to fair in two.

One important finding that enables
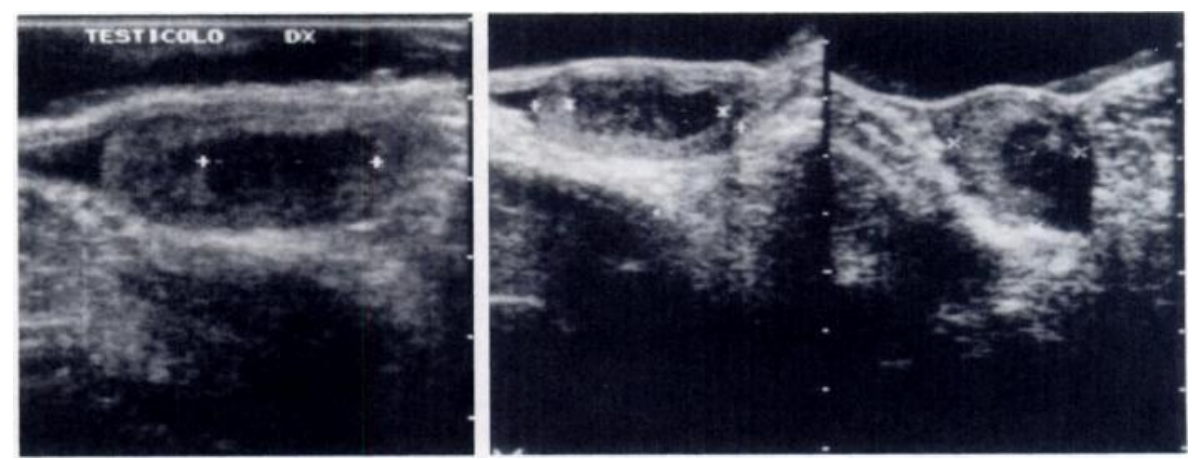

b.

Figure 5. Patient 3. (a) Longitudinal sonogram of the right testis and (b) longitudinal and axial sonograms of the left testis show bilateral multifocal hypoechoic lesions with sharp margins (cursors).

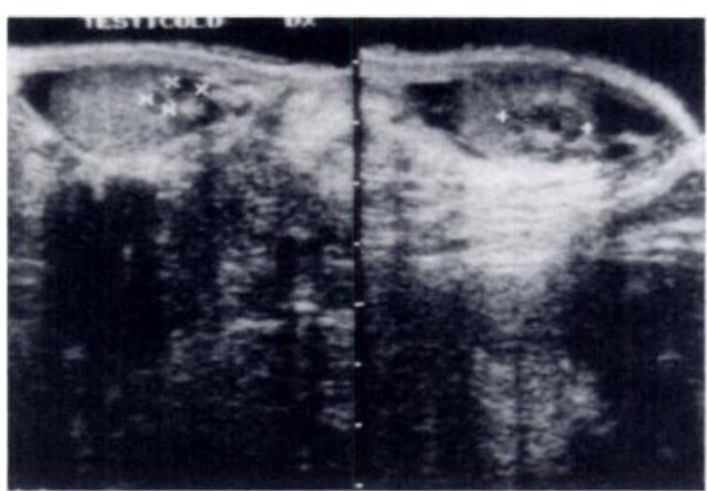

a.

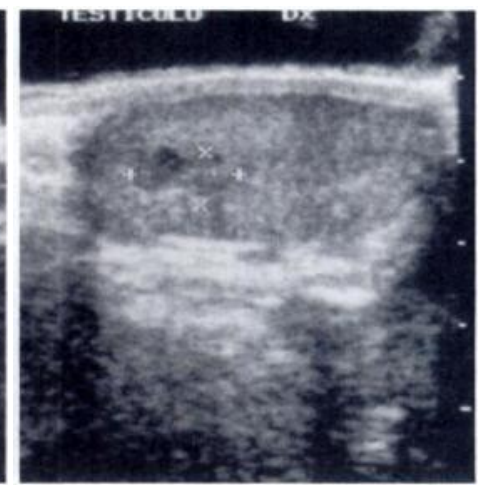

b.

Figure 6. Patient 2. (a) Oblique sonograms obtained in 1987 and (b) sagittal sonogram obtained in 1989 show the diameter of the testicular lesion (cursors) to be unchanged in this time period.

establishment of a correct diagnosis of TTAGS is the US pattern of the lesions. In our experience the nodules were always multifocal, were bilateral in six of eight patients, and were always hypoechoic. The margins were well defined in six patients. Testicular neoplasms, on the contrary, are frequently solitary lesions (10). When multifocal, neoplasms are located in only one testis: Bilateral multifocal testicular malignancy is rare (10).

The US aspects of the lesions in our patient population are similar to those described in the study of Seidenwurm et al (6), except that we did not find the acoustic shadowing pattern that was present in all their patients. This finding can be partially explained by the differences in the two populations. The mean age of the patients studied by Seidenwurm et al was 19.7 years, while the mean age of our patients was 15.6 years. Furthermore one of the patients studied by Seidenwurm et al had Addison disease. The sonographic pattern described by Willi et al (7) was similar to that observed in our patients.

At pathologic examination, the ma- jor lesion to be distinguished from TTAGS is the Leydig cell tumor, because the cells of both lesions look similar. However, this distinction can be determined histologically (4): At microscopic examination the abundant septa, composed of dense connective tissue, that surround lobules in TTAGS are rarely seen in Leydig cell tumors, which generally have thin septa composed of connective tissue. Moreover, the cytoplasm is more copious in TTAGS. Only 3\% of Leydig cell tumors are bilateral (18) compared with $75 \%$ of TTAGS in our study. Furthermore, Leydig cell tumors are usually seen in patients in their 3rd through 6 th decade compared with TTAGS seen in our patients in their 2nd decade. Finally, some bilateral "Leydig cell tumors" have proved to be TTAGS at careful endocrinologic investigation (19). None of our patients had Leydig cell tumors.

We did not assess the fertility of our patients because most of them were in their teens. Some authors (5) describe an association of TTAGS and poor fertility in patients with AGS. In some 
patients they report return of fertility and disappearance of the testicular masses after low-dose suppressive therapy with dexamethasone.

The presence of the clinical features of AGS is a major clue to the correct diagnosis. When bilateral, multifocal, hypoechoic testicular lesions are detected at scrotal US scanning, however, the possibility of AGS should be investigated, even in the absence of clinical symptoms. In fact, in one study of 40 patients with clinically detectable TTAGS (4), AGS was not diagnosed in $18 \%$ of the patients until the development of a testicular mass. Consequently, if multifocal and bilateral lesions are detected in a young boy, AGS should be kept in mind. Conversely, if multifocal testicular lesions are present in the testis of a patient with AGS (7), TTAGS are likely and frequent US follow-up is adequate for the diagnosis.

In conclusion, since most intratesticular lesions in young adults are malignant (10), it is important to recognize that bilateral, multifocal, hypoechoic nodules in the testes of patients with AGS are probably TTAGS. As the size of these lesions decreases or remains stable during hormonal therapy, further investigation may be limited to clinical and US follow-up. The greater sensitivity of
US compared with clinical evaluation in the detection of tiny multifocal lesions can help rule out malignancy; thus, testicular biopsy need be performed in only those patients with lesions that show a rapid increase in size.

\section{References}

1. Liddle GV. The adrenals. In: Williams $\mathrm{RH}$, ed. Textbook of endocrinology. 8th ed. Philadelphia: Saunders, 1982; 285-289.

2. Pang SY, Wallace NA. Worldwide experience in newborn screening for classica congenital adrenal hyperplasia due to 21-OH deficiency. Pediatrics 1988; 81:866874.

3. Miller WL, Levine L. Molecular and clinical advances in CAH. J Pediatr 1987; 11:117.

4. Rutgers LJ, Young RH, Scully RE. The testicular "tumor" of the adrenogenital syndrome. Am J Surg Pathol 1988; 12:503513.

5. Cutfield RG, Bateman JM, Odell WD Infertility caused by bilateral testicular masses secondary to congenital adrenal hyperplasia (21-hydroxylase deficiency). Fertil Steril 1983; 40:809-814.

6. Seidenwurm D, Smathers RL, Kan P, Hoffman A. Intratesticular adrenal rests diagnosed by ultrasound. Radiology 1985; 155:479-481.

7. Willi U, Atares M, Prader A, Zachmann M. Testicular adrenal-like tissue (TALT) in congenital adrenal hyperplasia: detection by ultrasonography. Pediatr Radiol 1991; 21:284-287.

8. Urban MD, Lee PA, Migeon CJ. Adult height and fertility in men with congenital virilizing adrenal hyperplasia. N Engl Med 1978; 299:1392-1396.

9. Shanklin DR, Richardson AP Jr, Rothstein G. Testicular hilar nodules in adrenogeni- tal syndrome: the nature of the nodules. Am J Dis Child 1963; 106:243-250.

10. Krone KD, Carroll BA. Scrotal ultrasound. Radiol Clin North Am 1985; 23:121-139.

11. Wilkins L, Fleishmann W, Hovard JE. Macrogenitosomia precox associated with hyperplasia of the androgenic tissue of the adrenal and death from corticoadrenal insufficiency. Endocrinology 1940; 26:385390.

12. Landling BH, Gold E. The occurrence and significance of Leydig cell proliferation in familial adrenal cortical hyperplasia. I Clin Endocrinol Metab 1951; 11:1436-1453.

13. Dahl EV, Bahn RC. Aberrant adrenal cortical tissue near the testis in human infants. Am J Pathol 1962; 40:587-598.

14. McDonald JH, Calams JA. A histological study of extraparenchymal Leydig-like cells. J Urol 1958; 79:850-858.

15. Glenn JF, Boyce WH. Adrenogenitalism with testicular adrenal rests simulating interstitial cell tumor. J Urol 1963; 89:456-463.

16. Prader A. Die cortisondauerbehandlung des kongenitalen adrenogenitalen syndroms. Helv Paediatr Acta 1953; 8:386-423.

17. Piyaratn P, Rosahn PD. Congenital adrenocortical hyperplasia associated with hyperplasia of aberrant (intratesticular) adrenal tissue. J Clin Endocrinol Metab 1957; 17:1245-1251.

18. Kim I, Young RH, Scully RE. Leydig cell tumors of the testis: a clinicopathological analysis of 40 cases and review of the literature. Am J Surg Pathol 1985; 9:177-192.

19. Miller EC Jr, Murray HL. Congenital ad renocortical hyperplasia: case previously reported as "bilateral interstitial cell tumor of the testicle." J Clin Endocrinol Metab 1962; 22:655-657. 